\title{
Measurement of vehicle ground speed with inertia sensors - computation issues
}

\author{
T. X. Mei \& H. Li \\ The University of Leeds, \\ School of Electronic and Electrical Engineering, Leeds, UK
}

\begin{abstract}
This paper proposes a new method for the measurement of train speed using bogie mounted inertial sensors, which may achieve a measurement accuracy far better than what is specified in UIC-5014-05 for wheel slide control. The proposed method measures the speed from a time shift between the dynamic motions of two wheelsets which are derived from the responses of a railway bogie to track excitations, in particular track irregularities that always exist in rail tracks. Two inertial sensors will be required and mounted onto a bogie frame to measure the bounce and pitch accelerations (or lateral and yaw accelerations). The measured signals will be processed via dedicated filters to produce estimated wheelset movements (i.e. the track irregularities). The principle of the proposed method will be presented, but the paper will be mainly focussed on the assessment of computational demand and impact on measurement performance.
\end{abstract}

Keywords: vehicle ground speed, indirect measurement, inertia sensors.

\section{Introduction}

The absolute speed of trains is one of essential parameters in railway operations and also essential for modern traction and braking control systems. Conventionally, the vehicle speed is measured indirectly from the rotational speed of wheels using encoders [5]. However the measurement accuracy of the technique is compromised by changes in wheel radius (due to wear, or lateral movement of the wheels) and limited resolution of the encoders acceptable for rail applications, but more critically the measurement may become completely unacceptable when wheel slip or slide occurs in acceleration (under traction) and deceleration (in braking) operation modes. A number of alternative 
measurement methods have been investigated, including the use of Doppler radars, eddy current sensors and video imaging [1-3]. Doppler radars are capable of measuring accurately train speeds at the positions/times of interest, but are not best suited for a continuous measurement which is essential in traction and braking control systems. The eddy current sensors or video imaging are proposed to detect time delays between two channels of measured signals, but there are practical limitations for their widespread application. In addition, it is possible to use GPS to derive vehicle speed from changes in train positions which could provide a simple solution if the reliability and availability of the signals can be ensured in all conditions.

This paper presents a study into the measurement of the vehicle absolute speed by detecting the time shift of track irregularities at two different wheelsets, typically the leading and trailing wheelsets of a bogie. Inertia sensors mounted on a bogie frame are required to measure the vibrations in the bounce and pitch directions, and then used to derive/estimate the track irregularities at the two wheelsets from the dynamic responses of the bogie to input excitations. The time shift between the two estimated track irregularities is then detected and converted into the speed measurement. The basic principle and general performance assessments of the proposed measurement technique are given in $[6,7]$, but this paper will be to examine main issues that affect the computational requirements such as the selection of step size for data sampling, the duration of running time windows, delays in the measurement, and detection reliability.

The paper is organised as follows. The configuration of a bogie vehicle used in the study and the principle of the proposed speed measurement method are introduced in section 2. The evaluation of the performance and robustness is provided in section 3 and practicalities such as measurement requirement and general issues related to computation demand are discussed in section 4 . Conclusions are given in section 5.

\section{Vehicle configuration and speed measurement principle}

Figure 1 shows a side view diagram of a conventional bogie vehicle used in the study, consisting of a vehicle body, two bogies and four wheelsets. Coil springs and linear dampers are modelled for the primary suspensions, where linearised models are used to approximate the properties of air-springs in the secondary suspensions. Only motions relevant to the study are considered, including the bounce and pitch movements of the car body and the two bogie frames. The external excitations to the vehicle considered in the study are (random) track irregularities due to track misalignment and are generated in the computer simulations to represent the roughness of a typical main line with an appropriate spatial power spectrum $\left(A_{r v} / f_{t}^{2}\right)$ for the track vertical displacement.

The new measurement technique is developed based on the observation that all wheelsets in a vehicle travel through any positions/sections of rail tracks consecutively with delays determined by the travelling speed and the distances between the wheelsets. As the wheel spacing is largely a fixed vehicle parameter, 


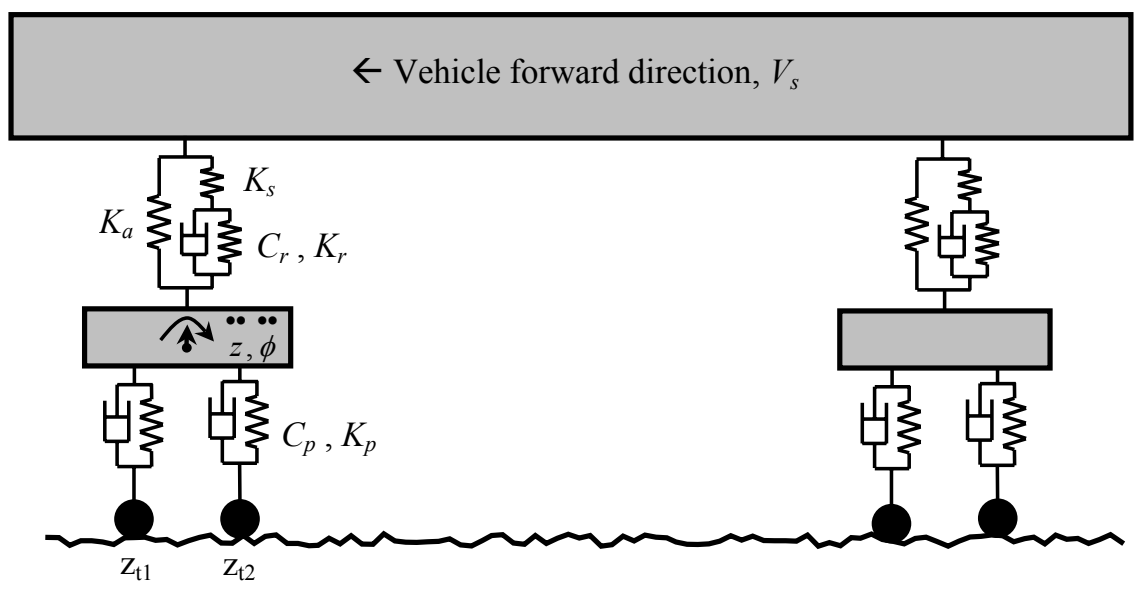

Figure 1: $\quad$ Side view of a conventional bogie vehicle.

there is clearly a direct link between the time shifts in wheelset responses to the same track excitations and the vehicle travelling speed.

Fig. 2 shows the key stages of the speed measurement scheme studied in this paper. Two inertia sensors are required to provide the signals for the bounce and pitch motions. Although it is possible to use two accelerometers mounted above the two suspensions, in practice it would be more convenient to use a single sensor box at/near the middle of the bogie frame to measure the bounce acceleration (from an accelerometer) and the pitch rate (from a gyro). The use of the bogie based sensors is preferred to a more direct approach of mounting sensors on wheelset axles, because of the issues related to the sensor range and reliability in a harsh operation environment.

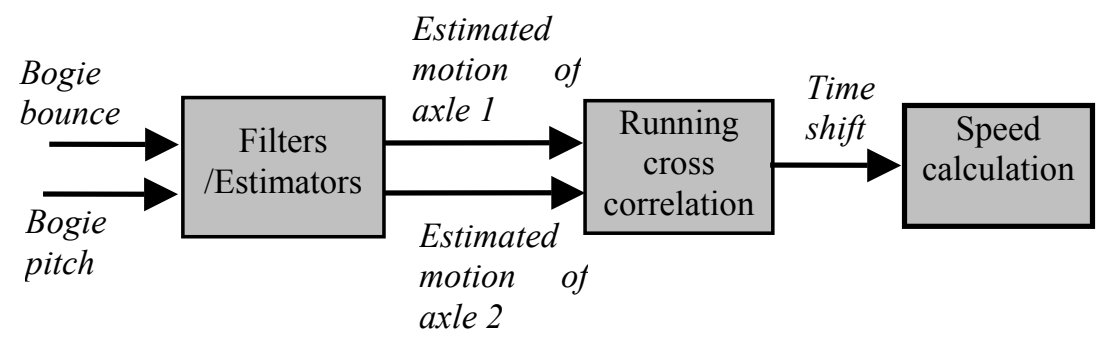

Figure 2: $\quad$ Speed measurement scheme.

The bounce and pitch motions are both affected by the inputs at the two wheelsets and hence the time delay (and the speed) from the leading wheelset to the trailing one can sometimes be detectable from the measured signals directly. However, the effect of the suspensions compromises the reliability, accuracy and speed range of the detection [6]. To overcome the problem, two filters are designed to estimate the track irregularities at the two wheelsets so that the effect 
of the bogie/suspension dynamics and associated uncertainties are removed. There have been studies on the use of model based techniques to estimate track inputs $[4,8]$, but for practical implementation it is also possible to use fairly simple filters to derive the signals accurate enough for detecting the time shifts between the two channels even though the absolute estimations may be less precise [7]. The detection of the time delay between the track irregularities (or any two signals) can be achieved by computing their cross correlations and detecting the time shift at peak values. The time shift can then be readily converted to the speed signal.

\section{Performance evaluation}

The UIC standard UIC-5014-05 for wheel slide control (in braking) defines specific requirements for the measurement of vehicle speed - relevant data for the measurement accuracy is given below:

- $\quad 5 \mathrm{~km} / \mathrm{h}(1.39 \mathrm{~m} / \mathrm{s})$, if measured speed is above the real speed

- $10 \mathrm{~km} / \mathrm{h}(2.78 \mathrm{~m} / \mathrm{s})$, if the real speed is lower than $200 \mathrm{~km} / \mathrm{h}$

- $15 \mathrm{~km} / \mathrm{h}(4.17 \mathrm{~m} / \mathrm{s})$, if the vehicle real speed is above $200 \mathrm{~km} / \mathrm{h}$

Those may be simplified to the measurement error of $5 \mathrm{~km} / \mathrm{h}(1.39 \mathrm{~m} / \mathrm{s})$ or less as a guideline for the performance assessment in the study.

Fig 3 shows the cross correlations between the estimated track irregularities at the vehicle speed of $60 \mathrm{~m} / \mathrm{s}$, where the sampling interval is $1 \mathrm{~ms}$. Three different

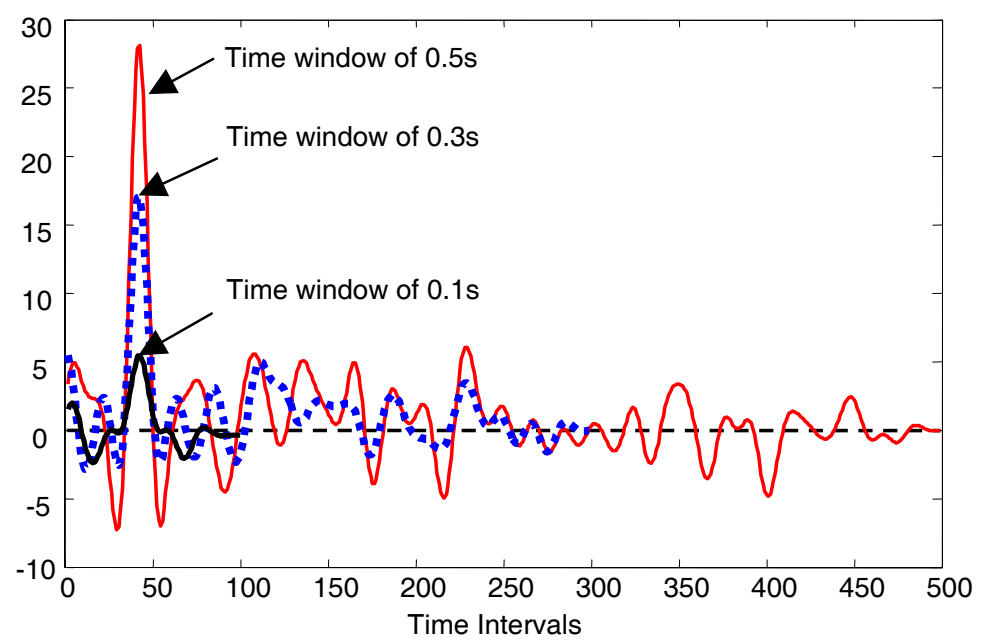

Figure 3: Cross correlations, at the speed of $60 \mathrm{~m} / \mathrm{s}\left(T_{s}=1 \mathrm{~ms}\right)$.

time windows of $0.1 \mathrm{~s}, 0.3 \mathrm{~s}$ and $0.5 \mathrm{~s}$ are used, and in all three cases a peak value is detected at the time shift $42 \mathrm{~ms}$ - indicating a measured speed of $59.52 \mathrm{~m} / \mathrm{s}$ and an measurement error of less than $0.5 \mathrm{~m} / \mathrm{s}$. The error is caused by the truncation in the 
detection of time shift. For the sampling interval of $1 \mathrm{~ms}$, the maximum error due to the truncation is $1.44 \mathrm{~m} / \mathrm{s}$ at the speed $(1.96 \mathrm{~m} / \mathrm{s}$ if the speed is $70 \mathrm{~m} / \mathrm{s})$. A smaller sampling interval will be needed to reduce the truncation error [9].

Although the selected time windows produce the same result in the time shift detection, there is a potential problem that the detection may become unreliable as a reduced time window tends to have relatively large cross correlations at other time shifts compared to the peak value. This is better demonstrated in Fig 4 where the cross correlations in all three cases are scaled to the same peak value. Therefore it is essential to determine and use appropriate time windows at different speeds and on different tracks, which may be ensured by monitoring a peak to mean ratio.

In general, longer time windows for cross correlation computations are required for detecting lower vehicle speeds because of the reduced signal frequencies in track irregularities. Fig. 5 shows the cross correlations of the estimated track inputs at the speed of $20 \mathrm{~m} / \mathrm{s}$ and Fig. 6 is the scaled version of Fig. 5. A time shift of $125 \mathrm{~ms}$ at the peak value is detected in all cases, which gives a measured speed of $20 \mathrm{~m} / \mathrm{s}$. There is no truncation error in this case, although there can be an error of upto $0.16 \mathrm{~m} / \mathrm{s}$ for the sampling interval of $1 \mathrm{~ms}$ in a worst case scenario.

Compared with the high speed of $60 \mathrm{~m} / \mathrm{s}$, the time windows have to be substantially increased in order to achieve similar level of peak/mean ratio. The increased time window may introduce additional measurement errors when a train is accelerated or decelerated. If the maximum accelerations of $0.5 \mathrm{~m} / \mathrm{s}^{2}$ (e.g. for inter-city trains) and $1 \mathrm{~m} / \mathrm{s}^{2}$ (e.g. for urban rapid transit) are considered, a time window of $1 \mathrm{~s}$ represents a typical delay of $0.5 \mathrm{~s}$ in time shift detection, or measurement error of $0.25 \mathrm{~m} / \mathrm{s}$ and $0.5 \mathrm{~m} / \mathrm{s}$ respectively.

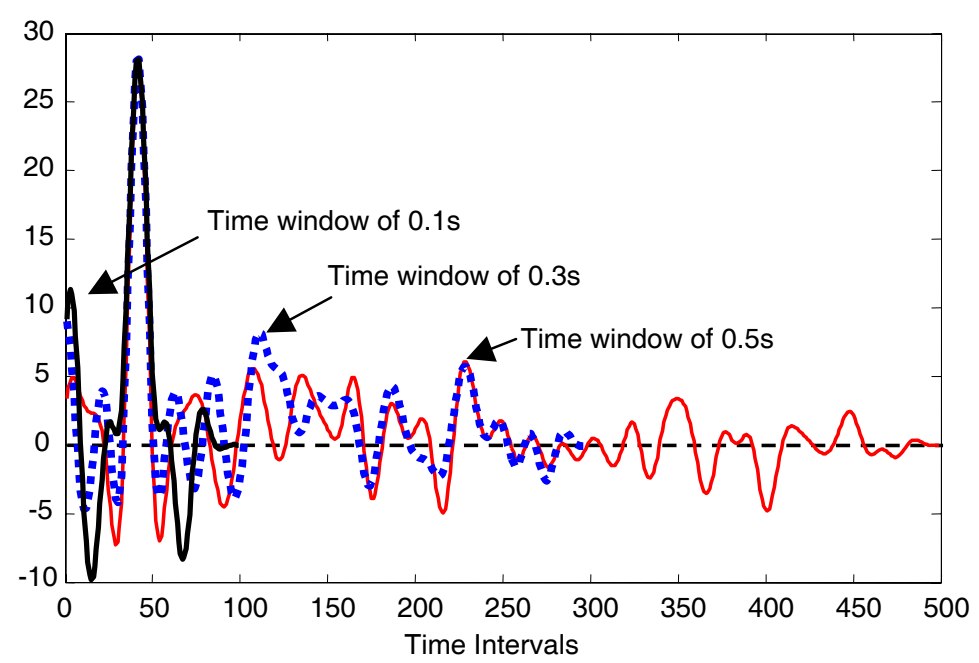

Figure 4: Scaled cross correlations, at the speed of $60 \mathrm{~m} / \mathrm{s}\left(T_{s}=1 \mathrm{~ms}\right)$. 


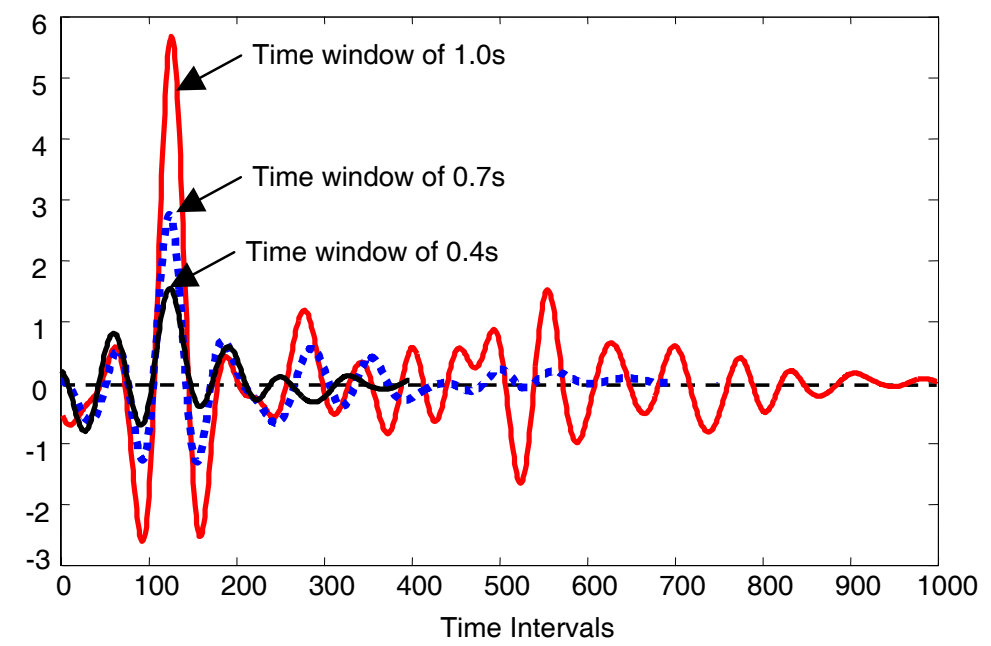

Figure 5: Cross correlations, at the speed of $20 \mathrm{~m} / \mathrm{s}\left(T_{s}=1 \mathrm{~ms}\right)$.

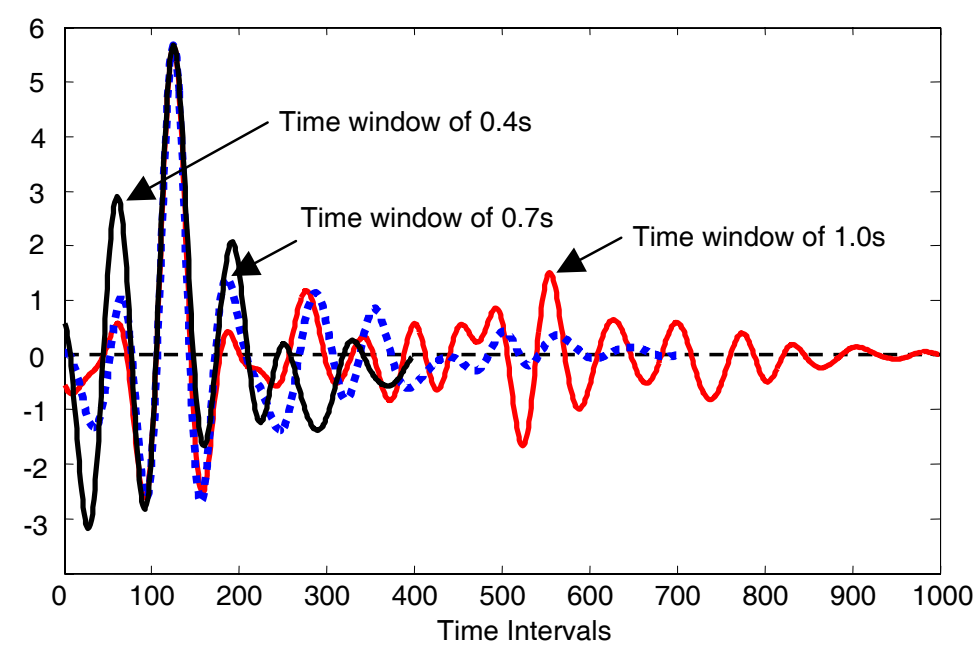

Figure 6: Scaled cross correlations, at the speed of $20 \mathrm{~m} / \mathrm{s}\left(T_{s}=1 \mathrm{~ms}\right)$.

The most challenging aspect of the proposed method is to push the detection boundary at the very low speed, where both the magnitude and the frequency of the track irregularities detectable from the bogie frame will be very low. Fig. 7 shows three cross correlations at the speed of $1.389 \mathrm{~m} / \mathrm{s}$ (or $5 \mathrm{~km} / \mathrm{h}$ ) with three different time windows of 5, 7 and 9s. The effect of the sensor noises (set at $1 \%$ of the maximum acceleration at the speed of $60 \mathrm{~m} / \mathrm{s}$ ) is clearly demonstrated, but 
the noises may not necessarily present a major problem in detecting the peak values [9]. Even with the noises, the detected time shifts in the three cases are $198 * 9 \mathrm{~ms}, 199 * 9 \mathrm{~ms}$ and $200 * 9 \mathrm{~ms}$ - giving the measured speed of $1.403,1.396$ and $1.389 \mathrm{~m} / \mathrm{s}$ respectively. The sampling interval used is $9 \mathrm{~ms}$, which would give a worst truncation error of $0.5 \%$.

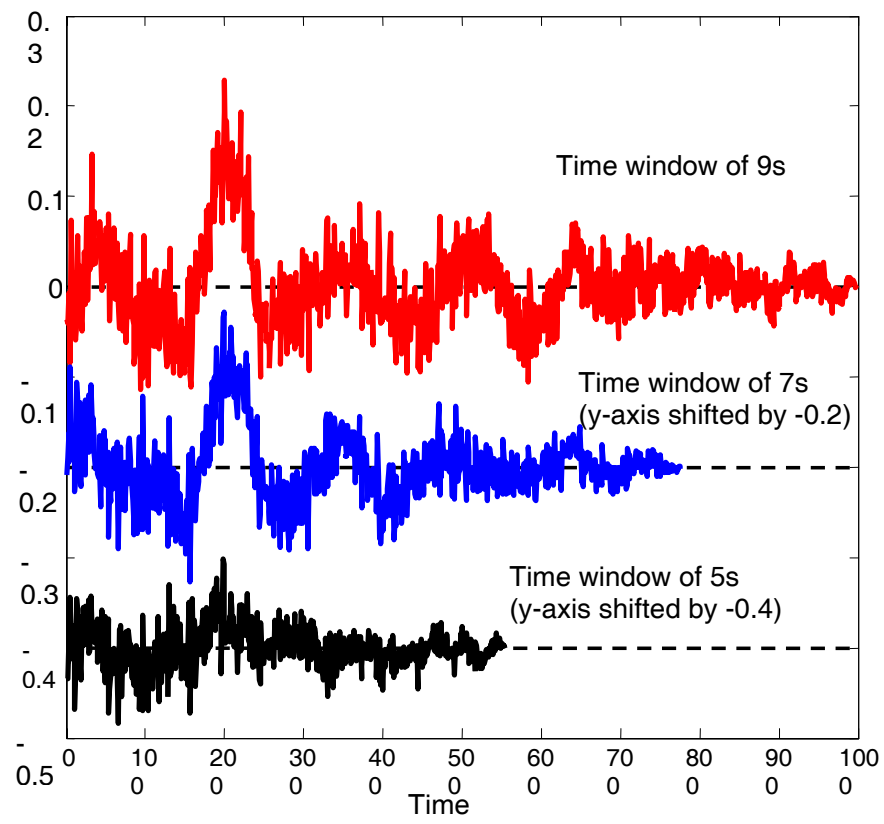

Figure 7: $\quad$ Cross correlations, at the speed of $1.389 \mathrm{~m} / \mathrm{s}\left(T_{s}=9 m s\right)$.

The more serious problem is that a much increased time window is needed at such low speed in order to detect the time shift between the two signals reliably (using the standard cross correlation calculations), which would result in not only substantial delay in the speed detection/updating, but also increased errors in acceleration/braking. Research is ongoing to study more intelligent data processing solutions to reduce the time for detecting the time shift and/or to compensate for the delay.

\section{Computation demand}

To implement the proposed method in real time for a continuous measurement of the vehicle speed, an on-line data processing using running time windows will need to be carried out in following steps (and repeated in a time interval of $T_{s}$ which may be varied on line).

A) Execution of two filters for the estimation of the track irregularities at the two wheelsets. Simple second order filters have been shown to be sufficient 
for the application [7]. The z-transform of the discrete time (digital) filters is given in equation 1 . The output signals from the filters are then re-combined to give the estimated track data. The required operations are: 12 Multiply; 5 Add and 5 Subtract operations for each time interval.

$$
G(z)=\frac{a_{0}+a_{1} z^{-1}+a_{2} z^{-2}}{1+b_{1} z^{-1}+b_{2} z^{-2}}
$$

B) Computation of the cross correlations $R_{x y}$ as shown in equation 2, where $v_{w 1}$ and $v_{w 2}$ are estimated derivatives of the track vertical displacements at the leading and trailing wheelsets respectively; $N$ is the total number of sampled data used for each time window $\left(T_{w d w}=N^{*} T_{s}\right)$ of the cross correlation computation; and $m$ is the number of shifted intervals. The time shift for each element of the resulted sequence $R_{x y}(m)$ will be $T_{s h f t}=m^{*} T_{s}$. This is the most computational intensive part of the data processing and in a worst case scenario could require $N^{2}$ number of operations for Multiply, Add/Subtract and Store/Read Data. It is possible (with more intelligent programming to reduce the operations to $N$ Multiply operations, $N$ Add and $N$ Subtract operations plus save and read data to/from memory $N$ times.

$$
R_{x y}(m)=\sum_{i=1}^{N} v_{w 1}(i+m) \cdot v_{w 2}(i)
$$

C) Detection of the time shift at the peak value $M$ and peak_square/mean_square ratio $P_{c}$. The former is used in the next step for speed calculation and the latter provides an indication of 'distinctiveness' of the peak value to ensure the reliability of the detection. The detection of the time shift $M$ may be achieved in $N$ number of data comparisons, although further improvement may be needed to reduce the effect of sensor noises at low speed by computing an average of the time shifts of several highest peaks. The computation of $P_{c}$ would involve $N+1$ Multiply, $N$ Add and 1 Divide operation(s) per time interval.

D) Calculation of the vehicle speed as indicated in equation 3 , where $2 L_{b}$ is the wheel spacing. The maximum computation will be 1 Divide operation per time interval. 1 Multiply operation would be needed whenever the time interval is changed, and some additional overhead in computation is also necessary to determine appropriate time intervals from previous/current speeds.

$$
V_{m}=\frac{2 L_{b}}{M \times T_{s}}
$$

Clearly, there is a high level of demand for computation. To evaluate the feasibility and cost implications for real applications of the measurement scheme, a medium spec DSP chip (at the cost of around US\$50) is selected - key performance parameters are given in Table 1. For a time window with 1000 data, the estimated computation time based on the device is less than $0.07 \mathrm{~ms}$ for single 
precision calculations and less than $0.12 \mathrm{~ms}$ for double precision operations. Both figures are much smaller than typical sampling intervals required for the truncation error considerations.

Table 1: $\quad$ Performance of TMS320C6413B (32 bits, 300MHz) floating point DSP - figures in bracket indicate clock cycles for pipelined instructions.

\begin{tabular}{|l|c|c|c|c|}
\hline & $\begin{array}{l}\text { Single } \\
\text { precision }\end{array}$ & $\begin{array}{l}\text { Double } \\
\text { precision }\end{array}$ & $\begin{array}{c}\text { Number of } \\
\text { Instructions in } \\
0.1 \mathrm{~ms}(\mathrm{SP})\end{array}$ & $\begin{array}{c}\text { Number of } \\
\text { Instructions in } \\
0.1 \mathrm{~ms}(\mathrm{DP})\end{array}$ \\
\hline Add & $3(1)$ & $6(2)$ & $10000,(30000)$ & $5000,(15000)$ \\
\hline Subtract & $3(1)$ & $6(3)$ & $10000,(30000)$ & $5000,(15000)$ \\
\hline Multiply & $3(1)$ & $6(2)$ & $10000,(30000)$ & $5000,(15000)$ \\
\hline Divide & 30 & 84 & 1000 & 357 \\
\hline If / then & 2 & 4 & 15000 & 7500 \\
\hline Load a word & 4 & & 7500 & \\
\hline Store a word & 1 & & 30000 & \\
\hline
\end{tabular}

\section{Concluding remarks}

A simple and practical method for the measurement of vehicle ground speed have been presented, which would be able to provide an effective measurement over a wide range of operation speeds and conditions including wheel slip/slide. The computation demand for real applications have been assessed, which indicate that the measurement scheme may be readily implemented using a low cost medium spec DSP device.

\section{References}

[1] Badmann R "Measuring vehicle ground speed with a radar sensor", Sensors, Vol 13, Issue 12, (1996).

[2] Engelberg $\mathrm{T}$ and Mesch F "Eddy current sensor system for non-contact speed and distance measurement of rail vehicles", 7th Intl Conf. on Computers in Railways, pp1261-1270, (2000).

[3] Harvey A and Cohen H "Vehicle speed measurement using an imaging method", IECON 1991, pp1730-1733, (1991).

[4] Kawasaki, J and Yousef-Toumi, K, "Estimation of rail irregularities", Proc. American Control Conference, Anchorage, USA, (2002).

[5] Kumagai N, Uchida S, Hasegawa I and Watanabe "Wheel slip rate control using synchronised speed pulse computing", Intl conf. on Computers in Railways, (2000).

[6] Mei, T X and Li, H "A novel approach for the measurement of absolute train speed”, Vehicle System Dynamics, Vol 46, supplement, (2008). 
[7] Mei, T. X. and Li, H., "Measurement of vehicle ground speed using bogie based inertial sensors", IMechE Proc, Part F - Rail and Rapid Transit, Vol 222, (2008)

[8] Mei, T.X., Li, H. and Goodall, R.M. "Kalman Filtering Applied to Actively Controlled Railway Vehicle Suspensions", Transactions of Institute of Measurement and Control, Vol. 23, No. 3, (2001)

[9] Mei, T.X and Li, H. "Measurement of vehicle ground speed using inertial sensors - accuracy and reliability", 4th IET Intl Conf. on Railway Condition Monitoring, Derby, UK, (2008). 